\title{
Inhaltsverzeichnis
}

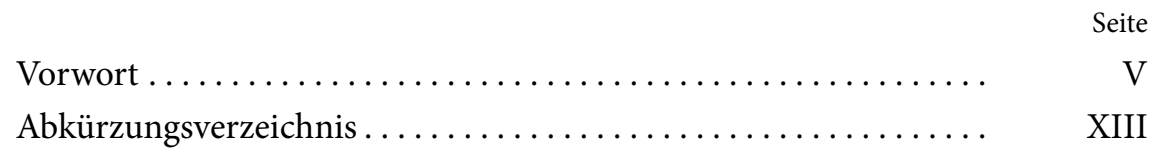

\section{Teil A}

Compliance

I. Betriebliche Datenschutzbeauftragte $\ldots \ldots \ldots \ldots \ldots \ldots \ldots \quad 5 \quad \begin{array}{ll}5 & 1\end{array}$

$\begin{array}{lll}\text { 1. Wann muss ein Datenschutzbeauftragter bestellt werden?..... } & 5 & 1\end{array}$

2. Was ist bei der Bestellung eines Datenschutzbeauftragten zu beachten? ................................ $12 \quad 3$

3. Welche Aufgaben und Befugnisse hat der Datenschutzbeauftragte?................................. $17 \quad 5$

II. Dokumentation und Folgenabschätzung (Vorabkontrolle). . $22 \quad 7$

4. Was wird aus den Verfahrensverzeichnissen?........... $22 \quad 7$

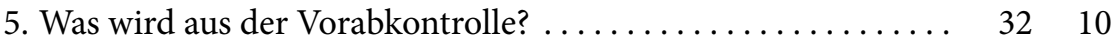

6. Wann ist eine Folgenabschätzung erforderlich? .......... $35 \quad 10$

7. Welche Regeln gibt es für den Ablauf der „Folgenabschätzung“? $42 \quad 12$

8. Was sind die möglichen Ergebnisse einer „Folgenabschätzung“? $45 \quad 12$

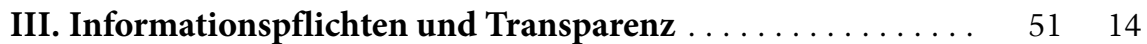

9. Welche Informationspflichten bestehen?............. $51 \quad 14$

10. In welcher Form sind die Informationen zu erteilen? ...... $61 \quad 18$

11. Welcher Zeitpunkt ist für die Informationen vorgeschrieben?. $\quad 68 \quad 20$

12. Gibt es Ausnahmen?.......................... $74 \quad 22$

IV. Allgemeine Datenschutzprinzipien und „risikobasierter Ansatz“ ............................... $81 \quad 24$

13. Welche Bedeutung hat der „Prinzipienkatalog"? .......... $84 \quad 24$

14. Welche Prinzipien umfasst der „Prinzipienkatalog“? ....... $87 \quad 26$

15. Welche Regelungen gibt es für „Privacy by Design“ und

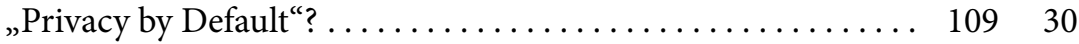

16. Was wird aus dem Grundsatz der Direkterhebung?......... $118 \quad 32$

17. An welchem Schutzgut orientieren sich die Datenschutzprinzipien? ............................. 12133

18. Wie ist der „risikobasierte Ansatz“ der DSGVO zu verstehen? . 12934 
19. Gegen welche Risiken der Datenverarbeitung wird der

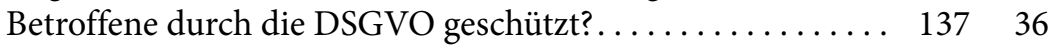

V. Technische und organisatorische Maßnahmen . . . . . . $140 \quad 37$

20. Welche Neuerungen gibt es bei der Datensicherheit? . . . . . 14237

21. Welche Neuerungen gibt es beim Datenschutz? . . . . . . . . 15139

VI. Pflichten bei Datenpannen . . . . . . . . . . . . . . . . . . . $154 \quad 40$

22. Welche Meldepflichten gibt es gegenüber den Aufsichtsbehörden?......................... 15641

23. Welche Meldepflichten gibt es gegenüber den Betroffenen?. . $162 \quad 42$

VII. Datentransfer in Drittstaaten . . . . . . . . . . . . . . . . . . $167 \quad 44$

24. Gibt es Änderungen beim „angemessenen Schutzniveau“? . . 17145

25. Gibt es Änderungen bei den Standardvertragsklauseln? . . . . 18148

26. Welche Neuerungen gibt es bei den „Binding Corporate Rules"?................................... 18949

27. Wann kommt eine Einzelgenehmigung durch die Aufsichtsbehörde in Betracht?....................... 19952

28. Welche Maßgaben gelten für die Einwilligung? . . . . . . . . 20253

29. Welche Auffangtatbestände gibt es? ............... 20554

VIII. Territorialer Anwendungsbereich der DSGVO _ . . . . . . $210 \quad 55$

30. Wird der territoriale Anwendungsbereich durch die DSGVO erweitert?....................... $217 \quad 57$

31. Wann liegt ein „Angebot“ an europäische Bürger vor? . . . . . 22258

32. Wann liegt eine „Beobachtung“ europäischer Bürger vor? . . 22459

33. Wann ist ein EU-Vertreter zu bestellen? ... . . . . . . . . . 22659

34. Was ist bei der Bestellung des EU-Vertreters zu beachten?. . 22960

35. Welche Pflichten hat der EU-Vertreter?... . . . . . . . . . 23060

IX. Haftung, Rechtsbehelfe, Sanktionen . . . . . . . . . . . 23261

36. Welche Haftungsrisiken gibt es nach der DSGVO? ....... . 23261

37. Welche Rechtsbehelfe hat der Betroffene? ............. 23662

38. Welche Bußgelder drohen nach der DSGVO?. . . . . . . . 24765

39. Nach welchen Kriterien richten sich die Bußgelder? ...... . 25166

40. Gibt es nach der DSGVO auch Straftaten?. . . . . . . . . . . 25567 


\section{Teil B}

\section{Was ändert sich an den Grundlagen des Datenschutzrechts?}

I. Sachlicher Anwendungsbereich der DSGVO...

41. Was wird aus dem Begriff der „personenbezogenen Daten“?... 26271

42. Was gilt für IP-Adressen, Cookies und andere „Kennungen“? . $279 \quad 74$

43. Gibt es noch Argumente für einen „relativen Personenbezug“? $281 \quad 74$

44. Gibt es Regelungen für anonyme Daten?............. $286 \quad 75$

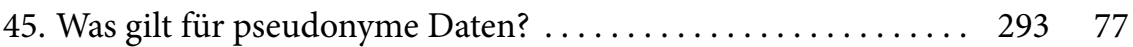

46. Gibt es noch eine „Haushaltsausnahme“? . . . ......... $306 \quad 79$

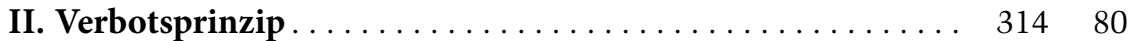

47. Bleibt es beim Verbotsprinzip? ........................ $314 \quad 80$

48. Welche Erlaubnistatbestände gibt es?................ 31981

49. Wofür gilt das Verbotsprinzip eigentlich? . . . . . . . . . . . . $324 \quad 82$

50. Gibt es Besonderheiten für den Schutz von Beschäftigtendaten? $333 \quad 85$

51. Was bedeutet das Verbotsprinzip für gesetzliche Verpflichtungen zur Datenverarbeitung?.................... 33786

52. Was bedeutet das Verbotsprinzip für die Kommunikationsfreiheit?............................... 34487

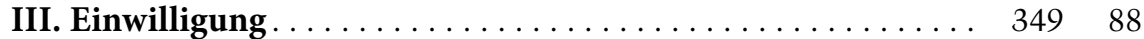

53. In welcher Form ist die Einwilligung zu erteilen? .......... $353 \quad 89$

54. Welche Anforderungen gelten für die „Informiertheit“? . . . . $367 \quad 92$

55. Können Einwilligungserklärungen Bestandteil von AGB sein?. $374 \quad 93$

56. Gibt es ein Kopplungsverbot? .................... $385 \quad 95$

57. Was gilt bei einem „klaren Ungleichgewicht“ zwischen den Beteiligten?................................ 39997

58. Welche Regelungen gibt es für den Widerruf von Einwilligungen?................................ 40398

59. Was gilt für die Einwilligung durch Minderjährige? ....... . $409 \quad 99$

IV. Vertrag als Erlaubnis. . . . . . . . . . . . . . . . . . . 416101

60. Was ändert sich bei der Verarbeitung von Kundendaten? .... 419102

61. Was gilt für Bestands- und Nutzungsdaten? . . . . . . . . . . 421102

V. Berechtigte Interessen . . . . . . . . . . . . . . . . . . . . . 422103

62. Gibt es einen allgemeinen Maßstab für „berechtigte

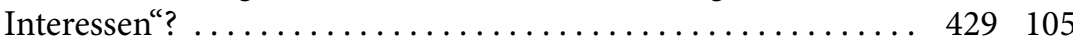


63. Welche Kategorien von „berechtigten Interessen“ lassen sich unterscheiden?......................... 438107

64. Gibt es Regelungen für die Datenverarbeitung zu präventiven Abwehrzwecken? ...................... 439108

65. Was gilt für die Videoüberwachung? ... . . . . . . . . . 447110

66. Welche Regelungen gibt es für das „Whistleblowing“? . . . . . 449110

67. Was gilt für allgemein zugängliche bzw. veröffentlichte Daten?.................................. 454112

68. Welche Regelungen gibt es für die Rechtsverfolgung und Rechtsverteidigung? ..................... 461113

69. Was gilt für die Werbung und den Adresshandel? . . . . . . . . 467114

70. Was gilt für den Datenaustausch innerhalb eines Konzerns? . 484118

71. Hat der Betroffene ein Widerspruchsrecht?. . . . . . . . . 492120

72. Welche Anforderungen gelten für das Widerspruchsrecht? . . 502122

VI. „Vereinbarkeit“ mit dem Erhebungszweck (Zweckänderung) . . . . . . . . . . . . . . . . . . . . . 509123

73. Ist der „vereinbare Zweck“ eine eigenständige Erlaubnisgrundlage? . . . . . . . . . . . . . . . . . . .

74. Unter welchen Voraussetzungen lässt sich eine „Vereinbar-

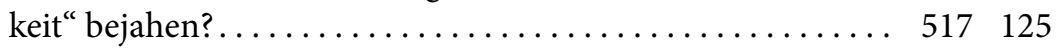

75. Welche Besonderheiten gibt es bei Einwilligungen?. . . . . . 521126

76. Kommt es bei „berechtigten Interessen“ zu einer „doppelten Abwägung"? ............................. 524126

77. Müssen die Betroffenen über „Zweckänderungen“ informiert werden?. .

VII. Gesundheitsdaten und andere besonders sensitive Daten . 528127

78. Für welche Daten gilt der besondere Schutz des Art. 9 DSGVO? ........................... 533128

79. Was versteht die DSGVO unter „Gesundheitsdaten“? . . . . . 535128

80. Gibt es besondere Regeln für das Gesundheitswesen? . . . . . 540129

81. Welche Regeln gelten für die Einwilligung? . . . . . . . . . 551132

82. Welche gesetzlichen Erlaubnisse gibt es? ............ . 554132

83. Welche Regeln gelten für die Verarbeitung von Daten über strafrechtliche Verurteilungen und Delikte? . . . . . . . 562135 
Teil C

Cloud Computing und Big Data

Rz. Seite

I. Auftragsverarbeitung . . . . . . . . . . . . . . . . . . . . . . 569137

84. Bleibt ausschließlich der Auftraggeber für die Datenverarbeitung verantwortlich? . . . . . . . . . . . . . . . . . . . 570138

85. Welche Pflichten treffen den Auftraggeber bei der Auswahl des Auftragsverarbeiters? ........................ 585141

86. Welche Änderungen gibt es bei Verträgen über die Auftragsdatenverarbeitung? ...................... 587141

87. Dürfen Subunternehmer eingeschaltet werden?. . . . . . . . . . 591144

II. Automatisierte Einzelentscheidungen . . . . . . . . . . . . . . $597 \quad 145$

88. Um welche Art von Verfahren geht es bei „Big Data“? . . . . . . 598147

89. Welche datenschutzrechtlichen Fragen können sich bei „Big Data“ stellen? ............................ $601 \quad 148$

90. Wie behandelt die DSGVO das „Profiling“? . . . . . . . . . . . . . 605149

91. Welche Regelungen gibt es für „automatisierte Entscheidungen im Einzelfall"? . . . . . . . . . . . . . . . . . . 611150

92. Gibt es Sonderregelungen für das Scoring? . . . . . . . . . . . . . 638155

93. Gibt es Sonderregelungen für Nutzungsprofile bei Telemedien?............................. $642 \quad 156$

\section{Teil D}

Betroffenenrechte, Datenschutzaufsicht und Selbstregulierung

I. Betroffenenrechte

649159

94. Welche allgemeinen Anforderungen gelten für die „Betroffenenrechte"? ................................... 651160

95. Welche Änderungen gibt es bei den Auskunftspflichten?... . . 669164

96. Welche Bedeutung hat das neue „Zugriffsrecht“? . . . . . . . . 675166

97. Gibt es Ausnahmen von den Auskunftspflichten und dem „Zugriffsrecht"? ...................... $678 \quad 166$

98. Welche Änderungen gibt es bei der Berichtigungspflicht? . . . 687168

99. Welche Änderungen gibt es bei den Pflichten zur Löschung? . 692169

100. Was bedeutet „Löschung" eigentlich genau? . . . . . . . . 701171

101. Welche Ausnahmen gibt es bei den Löschungsansprüchen? . . 702171

102. Wie sind die Ausnahmen im Einzelnen ausgestaltet? ... . . . . 704172

103. Für welche Fälle ist eine Sperrung von Daten vorgesehen? . . . $708 \quad 173$

104. Welche Pflichten hat der Datenverarbeiter bei einer Sperrung? 715175

105. Gibt es ein „Recht auf Vergessen“? . . . . . . . . . . . . 718175

106. Wie ist das Recht auf „Datenübertragbarkeit“ geregelt? . . . . 725177 
Rz. Seite

II. Datenschutzaufsicht . . . . . . . . . . . . . . . . . . . . . . . 734179

107. Welche Anforderungen stellt die DSGVO an die Datenschutzaufsicht?............................... 735179

108. Welche Aufgaben haben die Aufsichtsbehörden?... . . . . . . 740180

109. Welche Befugnisse haben die Aufsichtsbehörden?........ . 743180

110. Welchen Rechtsschutz gibt es?. . . . . . . . . . . . . . . 748182

111. Wann ist eine Aufsichtsbehörde „federführend“? . . . . . . . 750182

112. Wonach bestimmt sich die „Hauptniederlassung“ eines Unternehmens? ........................... 752183

113. Wann ist eine Aufsichtsbehörde „betroffen“? . . . . . . . . . . . 758184

114. Wie funktioniert das Abstimmungsverfahren unter den Aufsichtsbehörden?...................... 762185

115. Gibt es Ausnahmen von der Zuständigkeit der „federführenden“ Aufsichtsbehörde? . . . . . . . . . . . . . 767186

116. Welche Aufgaben hat der Europäische Datenschutzausschuss? $770 \quad 187$

117. Was wird aus dem Datenschutzbeauftragten der EU? . . . . . $773 \quad 187$

III. Regulierte Selbstregulierung: Verhaltensregeln und

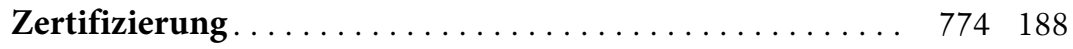

118. Was ändert sich bei den Verhaltensregeln? . . . . . . . . . . . 775188

119. Welche Möglichkeiten der Anerkennung von Verhaltens-

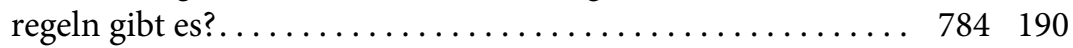

120. Welche Regelungen trifft die DSGVO für Zertifizierungen? . . 790191

121. Welche Vorteile haben ein Verhaltenskodex und eine Zertifizierung?........................ 801193

Stichwortverzeichnis ....................... 195 